\title{
Realization of Valley and Spin Pumps by Scattering at Nonmagnetic Disorders
}

\author{
Xing-Tao An, ${ }^{1,2}$ Jiang Xiao, ${ }^{3,4,5}$ M. W.-Y. Tu, ${ }^{1}$ Hongyi Yu, ${ }^{1}$ Vladimir I. Fal'ko, ${ }^{6}$ and Wang Yao ${ }^{1, *}$ \\ ${ }^{1}$ Department of Physics and Center of Theoretical and Computational Physics, University of Hong Kong, Hong Kong, China \\ ${ }^{2}$ School of Science, Hebei University of Science and Technology, Shijiazhuang, Hebei 050018, China \\ ${ }^{3}$ Department of Physics and State Key Laboratory of Surface Physics, Fudan University, Shanghai 200433, China \\ ${ }^{4}$ Collaborative Innovation Center of Advanced Microstructures, Nanjing 210093, China \\ ${ }^{5}$ Institute for Nanoelectronics Devices and Quantum Computing, Fudan University, Shanghai 200433, China \\ ${ }^{6}$ National Graphene Institute, University of Manchester, Booth St E, Manchester M13 9PL, United Kingdom
}

(Received 10 September 2016; revised manuscript received 28 November 2016; published 1 March 2017)

The recent success in optical pumping of valley polarization in two-dimensional transition metal dichalcogenides (TMDs) has greatly promoted the concept of valley-based informatics and electronics. However, between the demonstrated valley polarization of transient electron-hole pair excitations and practical valleytronic operations, there exist obvious gaps to fill, among which is the valley pump of longlived charge carriers. Here we discover that the quested valley pump of electrons or holes can be realized simply by scattering at the ubiquitous nonmagnetic disorders, not relying on any specific material property. The mechanism is rooted in the nature of the valley as a momentum space index: the intervalley backscattering in general has a valley contrasted rate due to the distinct momentum transfers, causing a net transfer of population from one valley to another. As examples, we numerically demonstrate the sizable valley pump effects driven by charge current in nanoribbons of monolayer TMDs, where the spin-orbit scattering by nonmagnetic disorders also realizes a spin pump for the spin-valley locked holes. Our finding points to a new opportunity towards valley spintronics, turning disorders from a deleterious factor to a resource of valley and spin polarization.

DOI: 10.1103/PhysRevLett.118.096602

The energy dispersion of electrons in crystalline solids usually has degenerate minima located at well-separated momentum space points, known as valleys. For low energy electrons, the valleys span an internal quantum degree of freedom, just like the spin. Similar to the potential use of spin polarization in spintronics, information can be represented by the valley polarization configurations, i.e., unequal population distribution among the degenerate valleys. Schemes to induce or manipulate valley polarization for potential valleytronics have been explored in various systems [1-12]. Unlike spintronic controls based on generic properties of spin, these valley control schemes rely on specific material properties such as valleydependent anisotropic dispersions [1,2], and carefully engineered strains [3,4], edges [5], or defects [6-10].

Two-dimensional hexagonal crystals such as graphene and monolayer transition metal dichalcogenides (TMDs) have newly emerged as a promising semiconducting platform for exploring valleytronic applications [13-21]. These materials have a time reversal pair of valleys spanning a pseudospin $1 / 2$, which can acquire spinlike properties that allow its manipulation similar to the spin controls. These include the valley Hall effect [13,15-19], the valley magnetic response [13,22-25], and the valley optical selection rules $[14,15]$. The latter, in particular, has enabled dynamic pumping of valley polarization of electron-hole pairs by circular polarized light in two-dimensional TMDs observed through the luminescence of these transient excitations [26-29], and the lifting of valley degeneracy via the valley-selective optical Stark effect [30]. These valley controls, however, rely on inversion symmetry breaking in the two-dimensional hexagonal lattices [31], which has limited the generalization to more platforms.

Here we discover a general mechanism to the pump valley based on its generic nature as a momentum space index, rather than extrinsically acquired properties dependent on host materials. Because of the distinct momentum transfers on a finite Fermi surface, intervalley backscattering by nonmagnetic disorder can have a valley-contrasted rate, which causes a net population transfer between the valleys. In a quasione-dimensional geometry, the valley current can be pumped out from both sides of a disordered region when a charge current is driven through. Remarkably the valley pump efficiency (i.e., per charge transmission) is shown to increase with the density of sharp disorders. For spin-valley coupled carriers, such a valley pump can also realize a spin pump. In monolayer TMDs, we numerically demonstrate the sizable valley pump of electrons by spin-independent scattering in zigzag nanoribbons, and the spin pump of holes by spin-orbit scattering in nanoribbons of various orientations. Our finding is an illuminating example on the advantage of exploiting the valley in future electronics, where the pseudospin controllability can directly arise from its momentum nature, in addition to those extrinsically acquired spinlike properties.

Consider a quasione-dimensional system where the energy dispersion has two well-separated valleys centered at finite momenta $K$ and $-K$. The disorder scattering for a 
carrier incident in valley $\tau(\tau= \pm K)$ can be characterized by the valley-conserved transmission $\left(T_{\tau, \tau}\right)$ and reflection coefficients $\left(R_{\tau, \tau}\right)$, and the valley-flip ones $\left(T_{\tau,-\tau}\right.$ and $\left.R_{\tau,-\tau}\right)$. If valley $\tau$ has $N_{\tau}$ sub-bands at the Fermi energy, $T_{\tau, \tau}\left(T_{\tau,-\tau}\right)$ and $R_{\tau, \tau}\left(R_{\tau,-\tau}\right)$, then refer to the overall valley-conserved (valley-flip) transmission and reflection summed over initial and final sub-bands of scattering.

The two valley-flip reflection coefficients $R_{K,-K}$ and $R_{-K, K}$ are different in general, as they correspond to intervalley scatterings with distinct momentum transfers, as illustrated in Fig. 1. In the first Born approximation, $R_{K,-K}$ and $R_{-K, K}$ correspond to different Fourier components of the scattering potential at $2 K+2 q_{F}$ and $2 K-2 q_{F}$, respectively, where $q_{F}$ is the Fermi wave vector. So large $q_{F}$ is favored to have a larger difference between $R_{K,-K}$ and $R_{-K, K}$. The two valley-flip transmission coefficients are always identical, i.e., $T_{K,-K}=T_{-K, K}$, as these two scattering channels are conjugates of each other (cf., Fig. 1). Thus, for a valley-unpolarized incident flux of $N$ electrons $\left(N=2 N_{K}=2 N_{-K}\right)$, the population difference between the $K$ and $-K$ valleys in the outgoing flux is (counting both transmission and reflection)

$$
P_{V} \equiv 2\left(R_{-K, K}-R_{K,-K}\right) .
$$

The scattering by the ubiquitous nonmagnetic disorder therefore provides a mechanism to the pump valley. (a)

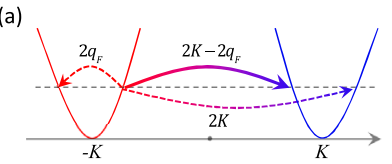

(b)
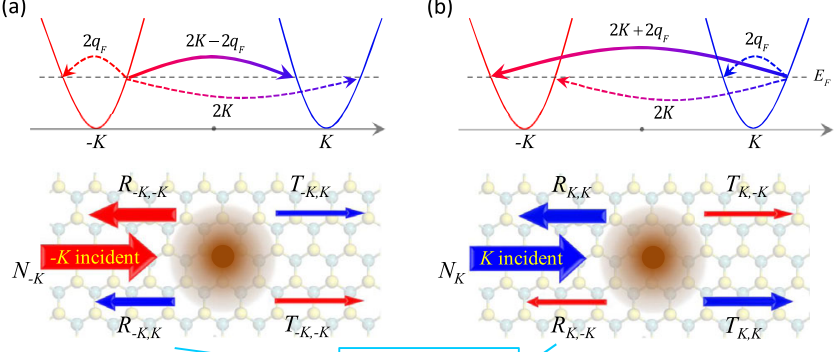

$\rightarrow P_{V}=2(R-K$,

(c)

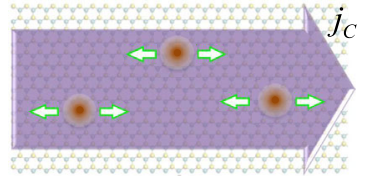

(d)

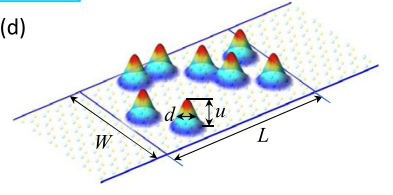

FIG. 1. (a) Schematics of the momentum transfers (upper) of the scattering channels (lower) for the electron incident in valley $-K$. (b) Electron incident in valley $K$. The valley-flip reflection coefficients $R_{-K, K}$ in (a) and $R_{K,-K}$ in (b) can differ because of the distinct momentum transfers (solid curved arrows in the upper panels), whereas the valley-flip transmission coefficients $T_{K,-K}$ and $T_{-K, K}$ are always equal. For an incident flux with one electron per valley, the disorder scattering thus transfers a net population of $R_{-K, K}-R_{K,-K}$ from valley $-K$ to $K$. (c) When a charge current $\left(j_{c}\right.$, purple arrow) is driven through, the valley current $\left(j_{V}\right.$, green arrows) is pumped out from both sides of the disorders. (d) Numerical demonstration of the valley pump uses monolayer $\mathrm{MoS}_{2}$ nanoribbons with disorders randomly distributed over a rectangular scattering region, where the pumped valley current is examined at the boundaries of the disordered region.
The pumped valley polarization is carried by both the reflection and transmission flux. If the $K$ and $-K$ valleys are time reversals of each other (as in the case of graphene and TMDs), the two valley-conserved reflection coefficients $R_{K, K}$ and $R_{-K,-K}$ are also identical. For a valleyunpolarized incident flux, the reflection flux then carries a valley current of $j_{V}=-\left(R_{-K, K}-R_{K,-K}\right) v_{F}$, while the valley current in the transmission flux is $j_{V}=\left(T_{K, K}-\right.$ $\left.T_{-K,-K}\right) v_{F}, v_{F}$ being the Fermi velocity. The valley currents on the two sides can be related through the sum rule $N_{\tau K}=T_{\tau K, \tau K}+R_{\tau K, \tau K}+T_{\tau K,-\tau K}+R_{\tau K,-\tau K}$. For the time reversal pair of valleys, this sum rule leads to

$$
T_{K, K}-T_{-K,-K}=-\left(R_{K,-K}-R_{-K, K}\right)=P_{V} / 2 .
$$

Namely, the valley current in the transmission flux has the same magnitude but opposite direction to that in the reflection flux [cf., Fig. 1(c)], and the net outward valley flow from the disorder is $P_{V} v_{F}$.

A disordered region can thus be exploited as a valley source when a charge current is driven through by bias voltage or temperature gradient [Fig. 1(c)]. The charge current, normalized by the thermodynamic driving force, is $j_{C}=T_{\text {sum }} v_{F}$ where $T_{\text {sum }} \equiv T_{K, K}+T_{-K,-K}+T_{K,-K}+T_{-K, K}$. The ratio between the outward valley flow from the disordered region and the charge current equals to $P_{V} / T_{\text {sum }}$, which characterizes the valley pump efficiency. It counts the valley population difference pumped per charge transmission.

If the incident flux has a valley polarization $\eta$, i.e., $(1+\eta) N / 2$ electrons in valley $K$ and $(1-\eta) N / 2$ electrons in $-K$, the overall effect of intervalley scattering then depends on $\eta$. The outgoing flux (reflection plus transmission) has an average valley polarization of $\eta+\Delta \eta$, where

$$
\Delta \eta=\frac{1}{N} P_{V}-\frac{1}{N} \Gamma_{V} \eta
$$

$\Gamma_{V} \equiv 2\left(R_{-K, K}+R_{K,-K}+T_{-K, K}+T_{K,-K}\right)$ here characterizes the valley depolarization by the intervalley scattering. The overall effect of intervalley scattering includes two counteracting terms: a valley pump term, and a depolarization term that is proportional to the incident valley polarization $\eta$. The competition between these two opposite effects determines whether the disorder scattering increases or decreases valley polarization of incident carriers.

As a remarkable feature of this valley pump mechanism, the pump efficiency is expected to increase with the number of sharp disorders in the scattering region, since each disorder pumps valley polarization of the same sign when the quantum interference between the multiple scatters can be neglected [see Fig. 1(c)]. The increase of $P_{V} / T_{\text {sum }}$ with the disorder number eventually saturates as suggested by the valley depolarization term in Eq. (3).

We demonstrate this valley pump in nanoribbons of monolayer TMDs. In these two-dimensional semiconductors, the conduction and valence band edges at the $\pm K$ 
valleys are contributed predominantly by the three metal $d$ orbitals [32]: $d_{z^{2}}, d_{x y}, d_{x^{2}-y^{2}}$. Our calculation is thus based on the tight-binding model constructed with these three orbitals [33],

$$
H=\sum_{i} \sum_{\mu} \varepsilon_{i \mu} c_{i \mu}^{\dagger} c_{i \mu}+\sum_{\langle i, j\rangle} \sum_{\mu \nu} t_{i \mu, j \nu} c_{i \mu}^{\dagger} c_{j \nu} .
$$

Here $c_{i \mu}^{\dagger}$ creates an electron on orbital $\mu$ at metal site $i$ in an orthogonal basis; the sums $\langle i, j\rangle$ run over all pairs of nearest-neighbor metal sites, and $t_{i \mu, j \nu}$ are the hopping integrals based on symmetry consideration (without the Slater-Koster two-center approximation) fitted from first principles band structures [33]. This simplified model describes well the low energy electrons and holes in the $\pm K$ valleys. Quantitative analysis can use more accurate tight-binding models accounting non-nearest-neighbor hopping [33] and more orbitals [34]. The spin-orbit coupling, being weak in the conduction bands [32], is neglected in discussing the valley pump of electrons.

The disorder potential is introduced by a position dependent on-site energy, $\varepsilon_{i \mu}=\varepsilon_{\mu}+u \sum_{l} \exp \left[-\left(\left|\mathbf{r}_{i}-\mathbf{r}_{l}\right|^{2}\right) /\left(2 d^{2}\right)\right]$, where $l$ runs over the disorders located at $\mathbf{r}_{l}$ randomly chosen from atomic sites in a region of length $L$ on a zigzag nanoribbon [cf., Fig. 1(d)]. All disorders are assumed with the same Gaussian profile of length scale $d=a$ ( $a$ is the lattice constant) and strength $u=-0.5 \mathrm{eV}$. The valleydependent scattering by the entire disordered region is calculated using a recursive Green's function technique [35]. The band parameters $\varepsilon_{\mu}$ and $t_{i \mu, j \nu}$ are taken from Ref. [33]. The periodic boundary condition is used at the ribbon edge to avoid the edge-state effects specific to this example of TMD nanoribbons, so that we can focus on generic aspects of the bulk valley pump that can be common to other materials. The presence of such edge states only has quantitative effects on the valley pump (cf., Supplemental Material [36,37]).

Figure 2(b) shows the calculated valley-dependent transmission and reflection for disorder scattering in a zigzag $\mathrm{MoS}_{2}$ nanoribbon. As expected, $T_{K,-K}=T_{-K, K}$ and $R_{K, K}=R_{-K,-K}$. The two valley-flip reflection coefficients $R_{K,-K}$ and $R_{-K, K}$ have a difference that increases with incident energy, and the same amount of difference is found between $T_{K, K}$ and $T_{-K,-K}$, as dictated by Eq. (2).

Valley pump per charge transmission is a figure of merit to quantify the efficiency of this pump scheme. As shown in Figs. 2(c) and 2(d), with the increase of disorder density, the overall charge transmission $T_{\text {sum }}$ decreases while the valley pump rate $P_{V}$ increases, both helping to enhance the valley pump efficiency. The valley pump per charge transmission $P_{V} / T_{\text {sum }}$ as a function of $n_{i}$ at a fixed Fermi energy is shown in Fig. 2(f). The pump becomes more efficient with the increase of disorder density. At $n_{i}=5 \%$, $P_{V} / T_{\text {sum }} \sim 30 \%$, meaning that equivalently three electrons are pumped out in full valley polarization per ten electrons transmitting through the disordered regions, which is a significant pump efficiency. At fixed disorder density,
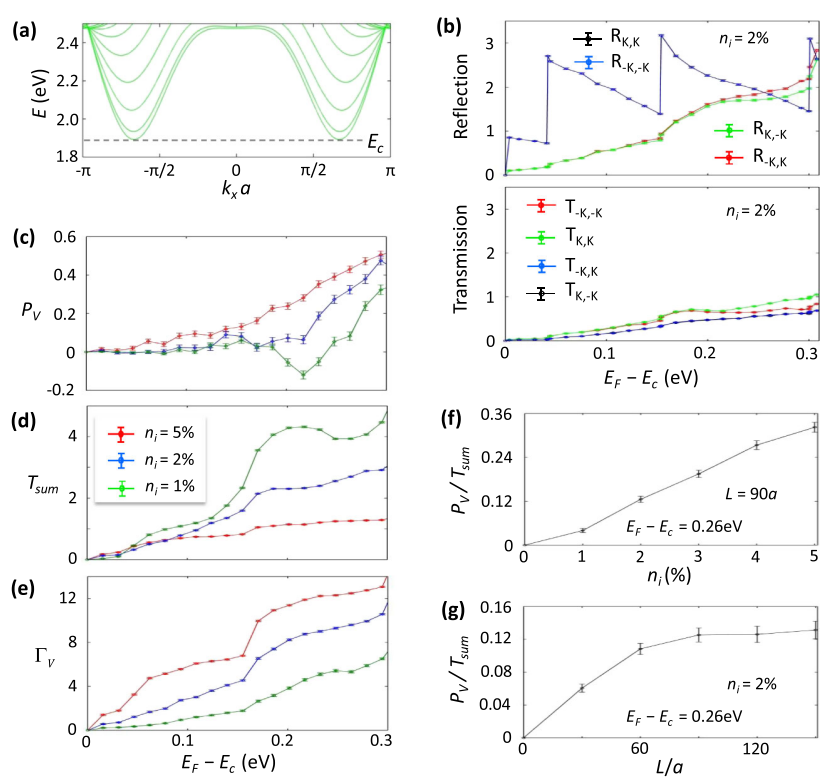

FIG. 2. (a) Conduction sub-bands from the tight-binding model without spin-orbit coupling. $E_{c}$ denotes the band edge. (b) The coefficients of intra and intervalley transmission and reflection by the disordered region [cf., Fig. 1(d)], as functions of the Fermi energy $E_{F}$, plotting the numbers of electrons scattered into the corresponding channels out of $N_{\tau}$ incident electrons in valley $\tau$, where $N_{\tau}$ is the number of sub-bands crossed by $E_{F}$ in valley $\tau$. (c) The valley pump rate $P_{V} \equiv 2\left(R_{-K, K}-R_{K,-K}\right)$, (d) the overall transmission $T_{\text {sum }} \equiv T_{K, K}+T_{-K,-K}+T_{K,-K}+T_{-K, K}$, and (e) the valley depolarization rate $\Gamma_{V} \equiv 2\left(T_{K,-K}+T_{-K, K}+R_{K,-K}+\right.$ $\left.R_{-K, K}\right)$. The length of the disordered region $L=90 a$ in (b)-(e), and $n_{i}$ is the disorder density. (f) The valley pump efficiency (i.e., per charge transmitted) $P_{V} / T_{\text {sum }}$ as a function of $n_{i}$, at $L=90 a$. (g) $P_{V} / T_{\text {sum }}$ as a function of $L$, at $n_{i}=2 \%$. $E_{F}-E_{c}=0.26 \mathrm{eV}$ in (f) and (g). The width of the zigzag nanoribbon is $W=27.7 a$ ( $a$ is lattice constant). In all plots, the dots represent calculated values averaged over 500 configurations of randomly generated disorder distributions, with the fluctuations shown as the error bar.

increasing the length $L$ of the scattering region can also enhance the pump efficiency, as shown in Fig. 2(g). The valley pump efficiency has the expected saturation behavior at large $L$, where the valley depolarization effect by the intervalley scattering starts to balance with the valley pump [cf., Eq. (3)].

Next we take into account the spin-orbit splitting in the Bloch bands. At the time reversal pair of valleys, the spin splitting must have opposite sign. This valley-dependent spin splitting effectively introduces a coupling between the spin and valley pseudospin. Therefore, even if the disorders are nonmagnetic, as long as they can flip spins, it is possible to pump spin. The idea can again be illustrated in the TMD nanoribbons, where the valance band edge has a spin splitting $\lambda$ of hundreds of $\mathrm{meV}$ [32]. So for band edge holes, the $K(-K)$ valley only has spin up (down) in out-of-plane direction; i.e., the spin index is locked to the valley index [cf., Fig. 3(a)]. We focus on this spin-valley locked energy window of $\lambda$, where the valley pump is a spin pump at the same time. 
(a)

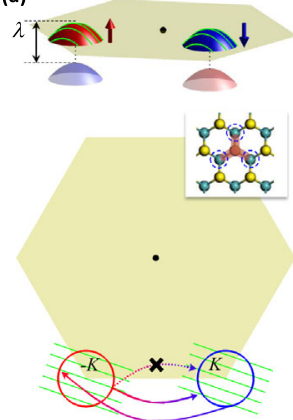

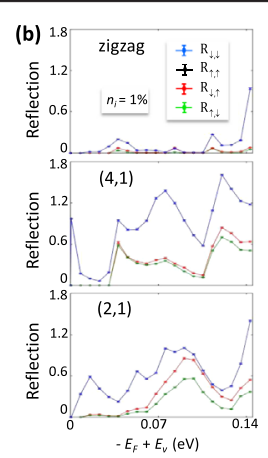

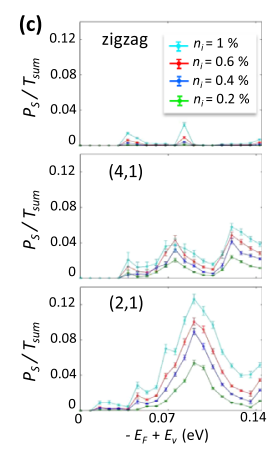

FIG. 3. (a) Valence band edges in monolayer TMDs with the valley-dependent spin splitting of magnitude $\lambda$. Red and blue denote spin up and down, respectively. Green line cuts of the twodimensional bands give the one-dimensional sub-bands of the nanoribbons (with periodic boundary condition). The two curved solid arrows denote the spin-flip reflections $R_{\uparrow, \downarrow}$ and $R_{\downarrow, \uparrow}$, which correspond to distinct momentum transfers on a finite Fermi surface with the spin-valley locking. The inset is the schematic of a nonmagnetic disorder with spin-flip hopping between three nearest-neighbor Mo sites. (b) Spin-conserved and spin-flip reflection coefficients calculated for monolayer $\mathrm{MoS}_{2}$ nanoribbons of zigzag, $(4,1)$ and $(2,1)$ orientations, respectively, plotting the numbers of holes scattered into the corresponding channels out of $N_{\uparrow(\downarrow)}$ incident holes in the spin $\uparrow(\downarrow)$ state, where $N_{\uparrow(\downarrow)}$ is the number of spin $\uparrow(\downarrow)$ sub-bands crossed by $E_{F}$. The disorder density $n_{i}=1 \%$. (c) The spin pump efficiency $P_{S} / T_{\text {sum }}$ at several disorder densities, where $P_{S} \equiv 2\left(R_{\uparrow, \downarrow}-R_{\downarrow, \uparrow}\right)$. In (b) and (c), the widths of zigzag, $(4,1)$ and $(2,1)$ nanoribbons are $27.7,27.5$, and $29.1 a$, respectively, and the lengths of the disordered regions are $90,79.4$, and 73.3 $a$, respectively. The dots represent averages over 500 configurations of randomly generated disorder distributions, with the fluctuations shown as the error bar.

Our numerical calculations are performed with the spinorbit coupling added to the three-band tight-binding model of monolayer TMDs [33],

$$
\begin{aligned}
H= & \sum_{i} \sum_{\mu} \varepsilon_{\mu} c_{i \mu}^{\dagger} c_{i \mu}+\lambda \sum_{i} \sum_{\mu, \nu} c_{i \mu}^{\dagger}\left(\mathbf{L}_{\mu, \nu} \cdot \mathbf{S}\right) c_{i \nu} \\
& +\sum_{\langle i, j\rangle} \sum_{\mu \nu} t_{i \mu, j \nu} c_{i \mu}^{\dagger} c_{j \nu}-i \alpha \sum_{\langle j, l\rangle_{d}} \sum_{\mu} c_{j \mu}^{\dagger}\left[\mathbf{S} \times \hat{\mathbf{r}}_{l j}\right]_{z} c_{l \mu},
\end{aligned}
$$

where the second term is the on-site interaction between orbital angular momentum $\mathbf{L}$ and $\operatorname{spin} \mathbf{S}$, which accounts for the strong spin-valley coupling in the valence band [33]. $\mathbf{L}_{\mu, \nu}$ denotes matrix element of $\mathbf{L}$ between orbital $\mu$ and $\nu$. For the disorder, we consider here a nonmagnetic one consisting of the spin-flip hopping between three nearest-neighbor metal sites [Fig. 3(a) inset]. Such hopping is described by the last term in Eq. (5), where $\hat{\mathbf{r}}_{l j}$ is the unit directional vector pointing from site $j$ to site $l,\langle j, l\rangle_{d}$ runs over the pairs of nearest-neighbor sites at each disorder, and $\alpha=0.5 \mathrm{eV}$.

In monolayer TMDs with the $D_{3 h}$ point group, the spin (valley) pump is symmetry allowed for nanoribbons with orientation other than the armchair. Figure 3(b) shows the calculated spin-flip and spin-conserved reflection coefficients, at a disorder density $n_{i}=1 \%$, in monolayer $\mathrm{MoS}_{2}$ nanoribbons of zigzag, (4,1) and $(2,1)$ orientations,

respectively. The intervalley reflection vanishes when the Fermi energy is between the edge of the first and the second sub-bands. This is because the intervalley reflection within the first sub-band is between a time reversal pair of states, which vanishes for a nonmagnetic disorder potential that preserves the time reversal symmetry [cf., Fig. 3(a)]. When the two spin-flip reflection coefficients $R_{\uparrow, \downarrow}$ and $R_{\downarrow, \uparrow}$ become finite at higher energy, their magnitudes are different, which leads to the spin pump.

Figure 3(c) shows $P_{S} / T_{\text {sum }}$, the spin pump per charge transmission, where $P_{S} \equiv 2\left(R_{\uparrow, \downarrow}-R_{\downarrow, \uparrow}\right)$. Note that Eqs. (1)-(3) can apply to the spin pump, with the valley index replaced by the spin index. For this disorder potential, both the reflection and the spin pump efficiency get stronger in directions other than the zigzag. The orientation dependence of the valley (spin) pump efficiency here is dominantly determined by the anisotropy of the disorders [cf., Fig. 3(a) inset], instead of the point group symmetry of the host lattice. In all three nanoribbons of different orientations, the spin pump gets more efficient with the increase of disorder density. We note that these nanoribbon orientations correspond to a distinct arrangement of the valleys with respect to the transport direction. The general requirement for this valley (spin) pump scheme to be applicable in the various materials is to have valleys with finite separation in the direction of transport, while their detail arrangement is not essential.

In summary, we have shown that the quested valley pump of electrons and holes can be realized with the ubiquitous nonmagnetic disorders. A new possibility for pumping spin is also made possible by scattering at nonmagnetic disorders in the presence of the spin-valley coupling. A practical source of valley (spin) polarization can thus be realized simply by passing charge current through a disordered region. The net effect of the disorder scattering is to turn a valley (spin) unpolarized incident current into valley (spin) polarized transmission and reflection. Valley and spin currents can then be extracted at the boundary of the disordered region [cf., Fig. 1(d)]. The spin pump effect is distinct in nature from spin generation schemes exploiting spin-dependent tunneling at semiconductor heterostructures [38-40], with the key difference being that the scatters are exploited here as spin sources (with net outward spin flow) instead of spin filters. It can be implemented in multivalley materials using various disorders that can cause intervalley spin flip.

It is interesting to compare this extraction scenario with the literature of current induced spin polarization homogeneously generated in infinite systems due to Rashba or Dresselhaus spin-orbit coupling [41]. The steady-state polarization in the homogeneous system has its magnitude limited by the ratio of the pump rate $P_{V}$ and the depolarization rate $\Gamma_{V}$ [cf., Eq. (3) and Supplemental Material [36]], which is typically small. In contrast, our valley (spin) pump scheme suggests utilizing the transmission or reflection current at the boundary of the disordered region, which has a valley (spin) polarization given by the ratio of the valley 
(spin) pump rate $P_{V}$ and the overall transmission $T_{\text {sum }}$ instead. Figures 2(c)-2(e) have shown that $P_{V}$ and $\Gamma_{V}$ increase while $T_{\text {sum }}$ decreases when the scattering is enhanced. Thus the valley and spin extraction efficiency at the boundary of the disordered region can be enhanced by increasing the disorder density, while the polarization established in a homogeneous system cannot. Moreover, here valley (spin) current is directly extracted into the clean regions on the two sides where valley (spin) lifetime is long with the absence of intervalley scattering.

This work is mainly supported by the Croucher Foundation under the Croucher Innovation Award, the Research Grants Council (Grant No. HKU17305914P) and University Grants Committee (Grant No. AoE/P-04/ 08) of Hong Kong SAR, and University of Hong Kong (ORA). X. A. is also supported by National Natural Science Foundation of China (Grants No. 11104059 and No. 61176089) and Natural Science Foundation of Hebei Province (Grant No. A2015208123). J. X. acknowledges support by Major State Basic Research Project of China (Grant No. 2014CB921600).

*wangyao@hku.hk

[1] O. Gunawan, Y. P. Shkolnikov, K. Vakili, T. Gokmen, E. P. De Poortere, and M. Shayegan, Phys. Rev. Lett. 97, 186404 (2006).

[2] Z. Zhu, A. Collaudin, B. Fauque, W. Kang, and K. Behnia, Nat. Phys. 8, 89 (2012).

[3] T. Low and F. Guinea, Nano Lett. 10, 3551 (2010).

[4] Z. Wu, F. Zhai, F. M. Peeters, H. Q. Xu, and K. Chang, Phys. Rev. Lett. 106, 176802 (2011).

[5] A. Rycerz, J. Tworzydlo, and C. W. J. Beenakker, Nat. Phys. 3, 172 (2007)

[6] D. Gunlycke and C. T. White, Phys. Rev. Lett. 106, 136806 (2011).

[7] I. Martin, Y. M. Blanter, and A. F. Morpurgo, Phys. Rev. Lett. 100, 036804 (2008).

[8] W. Yao, S. A. Yang, and Q. Niu, Phys. Rev. Lett. 102, 096801 (2009).

[9] F. Zhang, A. H. MacDonald, and E. J. Mele, Proc. Natl. Acad. Sci. U.S.A. 110, 10546 (2013).

[10] L. Ju, Z. Shi, N. Nair, Y. Lv, C. Jin, J. Velasco, Jairo, C. Ojeda-Aristizabal, H. A. Bechtel, M. C. Martin, A. Zettl, J. Analytis, and F. Wang, Nature (London) 520, 650 (2015).

[11] M. Shayegan, K. Karrai, Y. P. Shkolnikov, K. Vakili, E. P. De Poortere, and S. Manus, Appl. Phys. Lett. 83, 5235 (2003).

[12] K. Takashina, A. Fujiwara, S. Horiguchi, Y. Takahashi, and Y. Hirayama, Phys. Rev. B 69, 161304 (2004).

[13] D. Xiao, W. Yao, and Q. Niu, Phys. Rev. Lett. 99, 236809 (2007).

[14] W. Yao, D. Xiao, and Q. Niu, Phys. Rev. B 77, 235406 (2008).

[15] D. Xiao, G.-B. Liu, W. Feng, X. Xu, and W. Yao, Phys. Rev. Lett. 108, 196802 (2012).

[16] R. V. Gorbachev, J. C. W. Song, G. L. Yu, A. V. Kretinin, F. Withers, Y. Cao, A. Mishchenko, I. V. Grigorieva, K. S. Novoselov, L. S. Levitov, and A. K. Geim, Science 346, 448 (2014).
[17] M. Sui, G. Chen, L. Ma, W.-Y. Shan, D. Tian, K. Watanabe, T. Taniguchi, X. Jin, W. Yao, D. Xiao, and Y. Zhang, Nat. Phys. 11, 1027 (2015).

[18] Y. Shimazaki, M. Yamamoto, I. V. Borzenets, K. Watanabe, T. Taniguchi, and S. Tarucha, Nat. Phys. 11, 1032 (2015).

[19] K. F. Mak, K. L. McGill, J. Park, and P. L. McEuen, Science 344, 1489 (2014).

[20] Y. J. Zhang, T. Oka, R. Suzuki, J. T. Ye, and Y. Iwasa, Science 344, 725 (2014).

[21] H. Yu, Y. Wu, G.-B. Liu, X. Xu, and W. Yao, Phys. Rev. Lett. 113, 156603 (2014).

[22] A. Srivastava, M. Sidler, A. V. Allain, D. S. Lembke, A. Kis, and A. Imamoglu, Nat. Phys. 11, 141 (2015).

[23] G. Aivazian, Z. Gong, A. M. Jones, R.-L. Chu, J. Yan, D. G. Mandrus, C. Zhang, D. Cobden, W. Yao, and X. Xu, Nat. Phys. 11, 148 (2015).

[24] Y. Li, J. Ludwig, T. Low, A. Chernikov, X. Cui, G. Arefe, Y. D. Kim, A. M. van der Zande, A. Rigosi, H. M. Hill, S. H. Kim, J. Hone, Z. Li, D. Smirnov, and T. F. Heinz, Phys. Rev. Lett. 113, 266804 (2014).

[25] D. MacNeill, C. Heikes, K. F. Mak, Z. Anderson, A. Kormanyos, V. Zolyomi, J. Park, and D. C. Ralph, Phys. Rev. Lett. 114, 037401 (2015).

[26] H. Zeng, J. Dai, W. Yao, D. Xiao, and X. Cui, Nat. Nanotechnol. 7, 490 (2012).

[27] K. F. Mak, K. He, J. Shan, and T. F. Heinz, Nat. Nanotechnol. 7, 494 (2012).

[28] T. Cao, G. Wang, W. Han, H. Ye, C. Zhu, J. Shi, Q. Niu, P. Tan, E. Wang, B. Liu, and J. Feng, Nat. Commun. 3, 887 (2012).

[29] A. M. Jones, H. Yu, N. J. Ghimire, S. Wu, G. Aivazian, J. S. Ross, B. Zhao, J. Yan, D. G. Mandrus, D. Xiao, W. Yao, and X. Xu, Nat. Nanotechnol. 8, 634 (2013).

[30] E. J. Sie, J. McIver, Y.-H. Lee, L. Fu, J. Kong, and N. Gedik, Nat. Mater. 14, 290 (2015).

[31] X. Xu, W. Yao, D. Xiao, and T. F. Heinz, Nat. Phys. 10, 343 (2014).

[32] G.-B. Liu, D. Xiao, Y. Yao, X. Xu, and W. Yao, Chem. Soc. Rev. 44, 2643 (2015).

[33] G. B. Liu, W. Y. Shan, Y. G. Yao, W. Yao, and D. Xiao, Phys. Rev. B 88, 085433 (2013).

[34] S. Fang, R. Kuate Defo, S. N. Shirodkar, S. Lieu, G. A. Tritsaris, and E. Kaxiras, Phys. Rev. B 92, 205108 (2015).

[35] T. Ando, Phys. Rev. B 44, 8017 (1991).

[36] See Supplemental Material http://link.aps.org/supplemental/ 10.1103/PhysRevLett.118.096602 for effect of boundary condition, and steady-state polarization in homogeneous system, which includes Ref. [37].

[37] Y. Li, Z. Zhou, S. Zhang, and Z. Chen, J. Am. Chem. Soc. 130, 16739 (2008).

[38] S. A. Tarasenko, V. I. Perel, and I. N. Yassievich, Phys. Rev. Lett. 93, 056601 (2004).

[39] M. M. Glazov, P. S. Alekseev, M. A. Odnoblyudov, V. M. Chistyakov, S. A. Tarasenko, and I. N. Yassievich, Phys. Rev. B 71, 155313 (2005).

[40] Y. Y. Tkach, V. A. Sablikov, and A. A. Sukhanov, J. Phys.: Condens. Mat. 21, 125801 (2009).

[41] S. D. Ganichev, M. Trushin, and J. Schliemann, in Handbook of Spin Transport and Magnetism, edited by E. Y. Tsymbal and I. Zutic (Chapman and Hall/CRC, London, 2011), p. 487. 